\title{
The ratio of neutrophil to lymphocyte predicts interstitial lung disease and its prognosis in patients with primary Sjögren's syndrome: a retrospective analysis
}

\author{
Ke Zhang ${ }^{1}$, Wengen $\mathrm{Li}^{1}$, Chunmei $\mathrm{He}^{1}$, Xuechun $\mathrm{He}^{1}$, Jingyuan $\mathrm{Hou}^{2}$ \\ ${ }^{1}$ Department of Rheumatology and Immunology, Meizhou City People's Hospital, Meizhou, China; ${ }^{2}$ Scientific Research and Experiment Center, \\ Meizhou City People's Hospital, Meizhou, Meizhou, China \\ Contributions: (I) Conception and design: K Zhang, J Hou; (II) Administrative support: W Li; (III) Provision of study materials or patients: C He; \\ (IV) Collection and assembly of data: X He; (V) Data analysis and interpretation: K Zhang, J Hou; (VI) Manuscript writing: All authors; (VII) Final \\ approval of manuscript: All authors. \\ Correspondence to: Jingyuan Hou. Scientific Research and Experiment Center, Meizhou City People’s Hospital, 63 Huangtang Road, Meijiang District, \\ Meizhou 514031, China. Email: monkeyhjy@126.com.
}

Background During the course of disease, some patients with primary Sjögren's syndrome (pSS) develop
interstitial lung disease (ILD), which leads to a poor prognosis. There is currently a lack of methods to
identify high-risk patients with ILD and predict the prognosis. The purpose of this study was to analyze the
predictive value of the neutrophil to lymphocyte ratio (NLR) for the occurrence of ILD and its prognosis in
patients with pSS.

Methods: According to the inclusion and exclusion criteria, patients with pSS admitted to our hospital from May 2009 to November 2020 were included. The patients were divided into either an ILD group or a non-interstitial pneumonia [non-ILD (NILD)] group. We compared the baseline data of the two groups of participants, and the participants were followed up ( $\geq 1$ year) at clinic visits. Logistic multivariate regression analysis was used to analyze the risk factors related to ILD and prognosis in patients with pSS.

Results: A total of 217 patients with pSS were included, of which 71 (32.7\%) participants were diagnosed with ILD (ILD group) at the time of pSS diagnosis, and 146 (67.3\%) participants had no obvious ILD (NILD group). Based on follow-up results, logistic multivariate analysis revealed that NLR [relative risk $(\mathrm{RR})=1.81$, 95\% confidence interval (CI): 1.15 to 4.73 ], age ( $R R=1.43,95 \% \mathrm{CI}: 1.06$ to 3.66$)$, non-regular treatment (RR $=1.39,95 \%$ CI: 1.03 to 3.38$), \mathrm{ALB}<35 \mathrm{~g} / \mathrm{L}(\mathrm{RR}=1.32,95 \% \mathrm{CI}: 1.05$ to 3.17$)$, and elevated $\mathrm{CRP}$ ( $\mathrm{RR}=1.44$, 95\% CI: 1.10 to 4.53$)$ were associated with the occurrence of ILD in participants with pSS during followup. Age ( $\mathrm{RR}=1.28,95 \% \mathrm{CI}: 1.06$ to 2.25$)$, NLR ( $\mathrm{RR}=1.43,95 \% \mathrm{CI}$ : 1.12 to 2.57 ), non-regular treatment ( $\mathrm{RR}=1.51,95 \% \mathrm{CI}: 1.18$ to 3.01 ), and ILD ( $\mathrm{RR}=2.05,95 \% \mathrm{CI}: 1.36$ to 4.72 ) were related to all-cause death during follow-up.

Conclusions: The NLR is a risk factor for ILD in patients with pSS. The higher the NLR, the worse the prognosis; ILD significantly increases the risk of death in patients with pSS.

Keywords: Neutrophil to lymphocyte ratio (NLR); primary Sjögren's syndrome (pSS); interstitial lung disease (ILD); risk factors

Submitted Mar 24, 2021. Accepted for publication May 28, 2021.

doi: 10.21037/apm-21-1043

View this article at: http://dx.doi.org/10.21037/apm-21-1043 


\section{Introduction}

Previous studies have shown that systemic autoimmune diseases increase the risk of interstitial lung disease (ILD) (1). Primary Sjögren's syndrome (pSS) is a chronic autoimmune disease that can affect the entire system, mainly invading the exocrine glands and other organs outside the glands. The main clinical manifestations are dry mouth and eyes. There may also be symptoms of other system involvement, including fatigue, joint inflammation, myalgia, vasculitis, nephritis, lung disease, and other manifestations (2,3). The lung is a relatively common organ involved and the reported incidence is about $9-75 \%$ in pSS patients. Among them, ILD is a common type, and the prevalence of ILD in pSS patients is $3-11 \%$, putting patients at high risk of ventilatory disorders and secondary pulmonary hypertension. There are currently no radical cure methods. The main mitigation methods are: corticosteroids, immunosuppressants, and CD20 monoclonal antibodies.

Once ILD occurs, the prognosis of pSS patients is often poor. The quality of life (QOL) is significantly reduced, and the 5 -year mortality rate can increase by $16 \%$. The 10 -year overall mortality rate of pSS patients with ILD is 4 times of those patients without ILD (4-6). Therefore, it is particularly important to identify high-risk pSS patients with ILD as early as possible. Although high-resolution computed tomography (HRCT) can detect ILD early, it is not recommended in the guidelines to perform routine HRCT examinations on patients because of the exposure to radiation (7). In clinical practice, a simple, easy-to-use, lowcost, and less harmful identification method is needed. In recent years, the ratio of neutrophils to lymphocytes (NLR) has become a commonly used indicator of inflammation. It is used as a risk factor for many diseases, especially the identification and prognosis of inflammatory diseases $(8,9)$. Based on the results of previous studies, this study aimed to observe the value of NLR in predicting and prognosticating the occurrence of ILD in patients with pSS through retrospective analysis. We present the following article in accordance with the STROBE reporting checklist (available at http://dx.doi.org/10.21037/apm-21-1043).

\section{Methods}

\section{Study population}

In this study, patients with pSS admitted to our hospital from May 2009 to November 2020 were continuously included according to the inclusion and exclusion criteria. The inclusion criteria were as follows: (I) age $\geq 18$ years; (II) clear diagnosis of pSS; (III) complete medical history data, especially commonly used inflammation-related indicators, including blood cell count, C-reactive protein (CRP), and clear treatment records; (IV) HRCT examination or HRCT could be performed during this study; (V) the patients signed an informed consent form. The exclusion criteria were as follows: (I) malignant tumors; (II) other rheumatic diseases; (III) medical history data lacks key indicators; (IV) ILD had been diagnosed before pSS was diagnosed; (V) drugs that might cause ILD had been taken; (VI) heart failure. This study was approved by the Ethics Committee of Meizhou People's Hospital (2020-C102), and the study followed the Declaration of Helsinki (2013 revision) (10). All participants provided written informed consent.

\section{Study group}

The participants were allocated to either the ILD group or non-ILD (NILD) group based on their HRCT examination results. The diagnostic criteria for ILD were as follows (11-13): HRCT showing ground glass shadow, honeycomb shadow, consolidation shadow, lobular thickening shadow, central lobular nodule shadow, and grid shadow; clinical manifestations were dry cough, progressively worsening dyspnea, obvious after activity, with restrictive ventilation dysfunction and gas exchange disorder, while eliminating ILD caused by other diseases.

\section{Follow-up}

All patients were followed up for at least 1 year at clinic visits. The contents of follow-up included changes in pSS condition, treatment process, respiratory system symptoms and signs, and corresponding examination results. The simple lung function classification was used to assess the changes in the participant's respiratory function $(14,15)$ : Level 0: no breathing difficulties or cyanosis during moderate exertion; Level 1: breathlessness and mild cyanosis during moderate exertion; Level 2: breathlessness and mild cyanosis during mild exertion; Level 3: at rest, patient has difficulty breathing and severe cyanosis. The World Health Organization Quality of Life Instrument, Short Form (WHOQOL-BREF) scale was used to assess the participants' QOL $(16,17)$. 


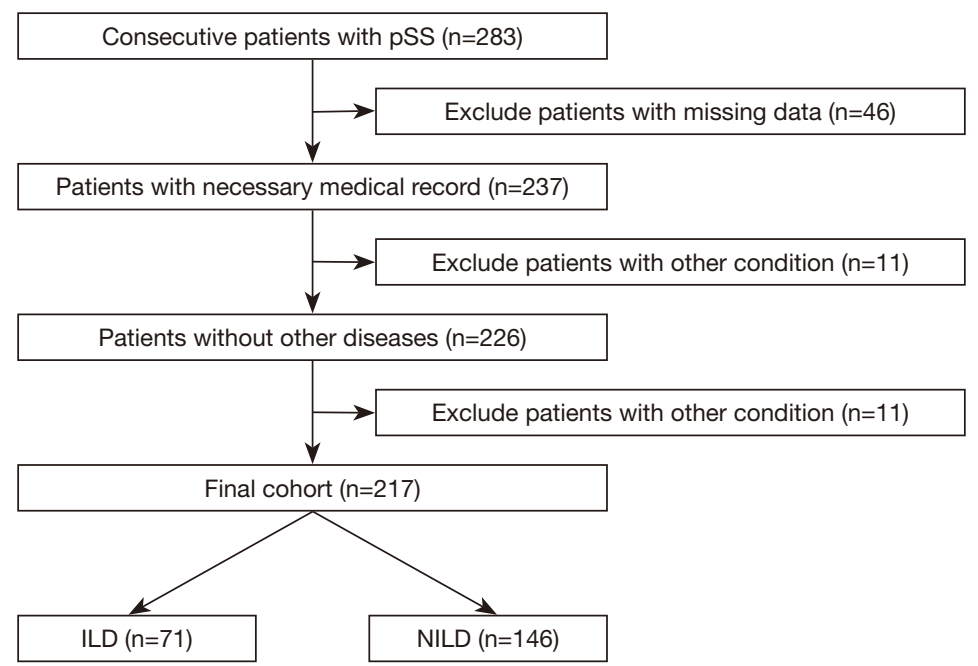

Figure 1 Study flowchart. Other condition: (I) malignant tumors; (II) other rheumatic diseases; (III) the history data lacks key indicators; (IV) ILD has been diagnosed before pSS is diagnosed; (V) drugs that may cause ILD are taken; (VI) heart failure. ILD, interstitial lung disease; pSS, primary Sjögren's syndrome; NILD, non-interstitial lung disease.

\section{Data collection}

The clinical data of the participant at the first HRCT after the diagnosis of pSS were collected, including demographic data, blood test results, HRCT results, drug treatment plan, and follow-up results. The data was drawn from the participant's medical records during hospitalization, and outpatient medical records (that is, follow-up records). The primary endpoint was death from all causes, and secondary endpoints included QOL, and newly diagnosed ILD. In order to eliminate the bias caused by data collection, data collectors and data analysts were unaware of the research purpose and plan. Patients with incomplete data were excluded according to the inclusion and exclusion criteria.

\section{Statistical analysis}

The software SPSS version 22.0 (IBM, Chicago, IL, USA) was used for statistical analysis. When the continuous variable conformed to the normal distribution, it was represented by the mean \pm standard deviation $(\mathrm{SD})$, and the Student $t$-test was used for comparison between groups; when it did not conform to normal distribution, it was represented by the median and quartiles, and the rank sum test was used for the comparison between groups. The qualitative data were represented by $\mathrm{n}(\%)$, and the comparison between groups was performed using chisquare test or Fisher's exact test. Logistic regression was used to analyze the relationship between each factor and the primary and secondary endpoints, namely major adverse events (MAEs). A P value $<0.05$ indicated a statistically significant difference.

\section{Results}

\section{Comparison of baseline data of participants in the ILD group and NILD patients}

According to the inclusion and exclusion criteria (Figure 1), a total of 217 patients with pSS were included in this study, of which $71(32.7 \%)$ participants were diagnosed with pSS and were also found to have complicated ILD (ILD group), and 146 patients $(67.3 \%)$ had no obvious ILD (NILD group). Comparison of baseline data between the two groups found statistical differences in factors including age, NLR, and treatment (Table 1).

\section{Follow-up outcome}

In this study, 217 pSS participants were followed up for 1-18 years, with a median follow-up of 8 [4-12] years. The ILD group was followed up for 8 [5-11] years, the NILD group was followed up for 8 [4-12] years, and there was no statistical difference in follow-up duration $(\mathrm{u}=0.376$, $\mathrm{P}=0.707$ ). During the follow-up period, 18 new ILD cases $(8.3 \%)$ were detected in 105 participants who were 
Table 1 Comparison of baseline data between ILD group and NILD patients

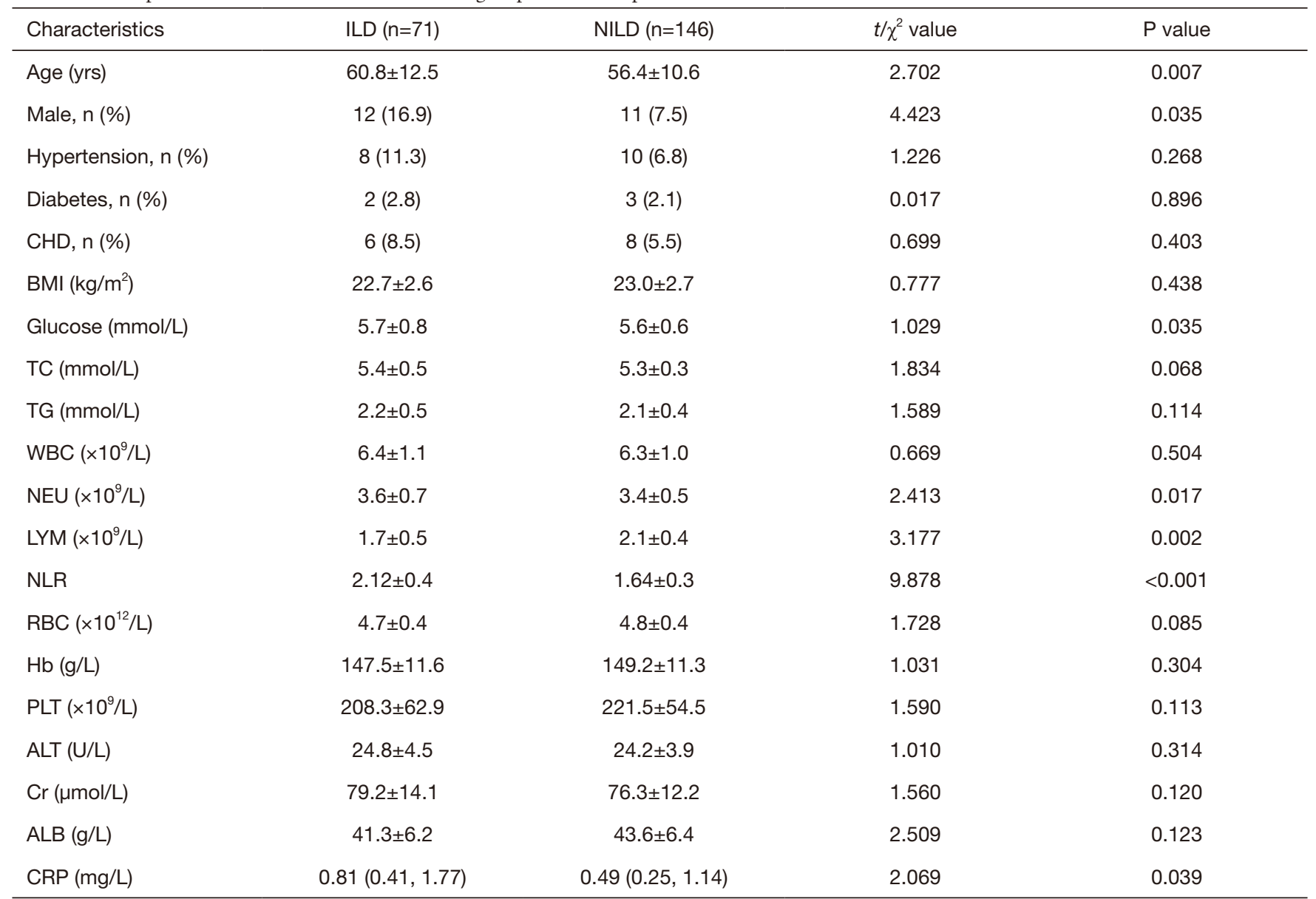

ILD, interstitial lung disease, NILD, non-interstitial lung disease; CHD, coronary heart disease; BMI, body mass index; TC, total cholesterol; TG, triglyceride; WBC, white blood cell; NEU, neutrophil; LYM, lymphocyte; NLR, neutrophil to lymphocyte ratio; RBC, red blood cell; Hb, hemoglobin; PLT, platelet; ALT, alanine transaminase; Cr, creatine; ALB, albumin; CRP, C-reaction protein.

rechecked by HRCT. In total, 89 cases $(41.0 \%)$ with ILD were identified in this study. A total of 35 cases (16.1\%) died during follow-up. The QOL score showed that the QOL of participants in the NILD group was higher than that in the ILD group. Participants in the NILD group had better lung function (Table 2).

\section{Analysis of factors related to ILD in patients with pSS}

Logistic multivariate analysis showed that NLR, age, nonregular treatment, albumin (ALB) $<35 \mathrm{~g} / \mathrm{L}$, CRP (Table 3), and so on, were related to the presence of ILD in participants with pSS. In the 1-18 years of follow-up of 146 participants with pSS without ILD, 105 underwent reHRCT examination, and 18 (17.1\%) had ILD detected (Figures 2,3). A multivariate analysis of this result found that baseline NLR, ALB $<35 \mathrm{~g} / \mathrm{L}$, CRP, age, and non-regular treatment were risk factors for the occurrence of ILD in pSS patients during follow-up (Table 4).

\section{Analysis of factors related to the prognosis of pSS patients}

Logistic regression analysis of the main adverse events during the follow-up period of pSS patients found that age, NLR, non-regular treatment, and ILD were closely related to all-cause death (Table 5).

\section{Discussion}

The retrospective analysis of this study found that 71 cases of ILD (32.7\%) were detected when pSS was first diagnosed among 217 patients, and 18 cases of ILD were 
Table 2 Comparison of follow-up results between participants in ILD group and NILD patients

\begin{tabular}{|c|c|c|c|c|}
\hline Outcomes & ILD $(n=71)$ & $\operatorname{NILD}(n=146)$ & Statistical value & $P$ value \\
\hline All-cause death, n (\%) & $17(23.9)$ & $18(12.3)$ & 4.764 & 0.029 \\
\hline Respiratory death, n (\%) & $15(21.1)$ & $10(6.8)$ & 9.553 & 0.002 \\
\hline RF hospitalization, n (\%) & $26(36.6)$ & $19(13.0)$ & 16.195 & 0.0001 \\
\hline WHOQOL-BREF score & $58.5 \pm 11.8$ & $63.6 \pm 12.4$ & 2.887 & 0.004 \\
\hline Lung function, $\mathrm{n}(\%)$ & & & 14.665 & 0.002 \\
\hline I & $25(35.2)$ & $69(47.2)$ & & \\
\hline II & $24(33.8)$ & $61(41.8)$ & & \\
\hline
\end{tabular}

ILD, interstitial lung disease; NILD, non-interstitial lung disease.

Table 3 Analysis of factors related to the presence of ILD in patients with pSS

\begin{tabular}{llll}
\hline Factors & RR & $95 \%$ Cl & P value \\
\hline NLR & 2.36 & $1.29-5.01$ & 0.006 \\
Age & 1.85 & $1.14-4.28$ & 0.029 \\
Not regular treatment & 2.02 & $1.09-4.59$ & 0.014 \\
ALB $<35 \mathrm{~g} / \mathrm{L}$ & 1.48 & $1.05-2.33$ & 0.032 \\
CRP & 1.64 & $1.18-4.97$ & 0.025
\end{tabular}

ILD, interstitial lung disease; pSS, primary Sjögren's syndrome; RR, relative risk; Cl, confidence interval; NLR, neutrophil to lymphocyte ratio; ALB, albumin; CRP, C-reactive protein.

newly diagnosed during the 1-18 years follow-up period (accounting for $17.1 \%$ of all participants who underwent HRCT during the follow-up period), which made a total of 89 cases (accounting for $41.0 \%$ of the total cases). The detection rate was basically the same as that of other studies $(6,18)$. Our follow-up results showed that the prognosis of patients with ILD at the first diagnosis of pSS was significantly worse than that of patients without ILD. The main manifestation was that the incidence of adverse events in these patients during the follow-up period was significantly higher than that of NILD participants, and their QOL was poorer. Multivariate analysis found that multiple factors were related to pSS patients with ILD and its prognosis, including age, NLR, non-regular treatment, $\mathrm{ALB}$, and CRP. At the same time, ILD significantly increased the risk of poor prognosis.
Rheumatic diseases are systemic inflammations that often involve lung tissues, resulting in decreased lung compliance and thickening of respiratory membranes, leading to restrictive ventilatory and gas exchange disorders. Patients often manifest asthma after exercise, stubborn hypoxemia, and eventually respiratory failure occurs $(19,20)$. Early targeted treatment can delay progression of the disease and improve the patient's respiratory function and QOL $(21,22)$. The key point is to diagnose high-risk patients and lung changes at the early stage. Unfortunately, there have been relatively few related studies. Moreover, such studies are often cross-sectional studies, mainly describing that certain factors are statistically related to the presence of ILD in pSS patients at a certain point in time. In a metaanalysis (23), He et al. examined 23 studies, including 6,157 patients with pSS, and found that the detection rate of ILD 


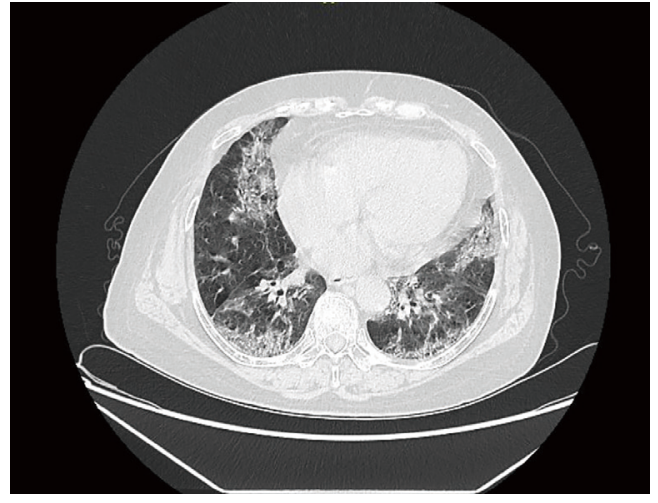

Figure 2 Case 1. Pulmonary manifestation of diffused ILD in a participant with pSS. ILD, interstitial lung disease; pSS, primary Sjögren's syndrome.

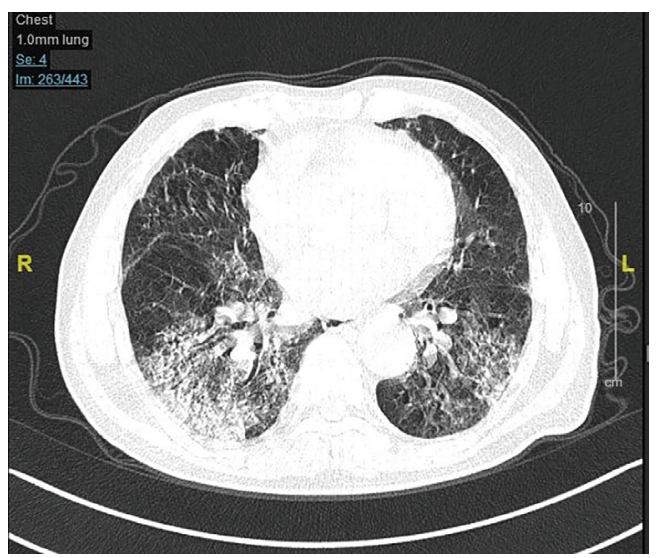

Figure 3 Case 2. Pulmonary manifestation of diffused ILD, especially lower lobe of double lung in a participant with pSS. ILD, interstitial lung disease; pSS, primary Sjögren's syndrome.

Table 4 Analysis of related factors of ILD occurrence in patients with pSS

\begin{tabular}{lccc}
\hline Factors & $\mathrm{RR}$ & $95 \% \mathrm{Cl}$ & $\mathrm{P}$ value \\
\hline NLR & 1.81 & $1.15-4.73$ & 0.008 \\
Age & 1.43 & $1.06-3.66$ & 0.029 \\
Not regular treatment & 1.39 & $1.03-3.38$ & 0.014 \\
ALB $<35 \mathrm{~g} / \mathrm{L}$ & 1.32 & $1.05-3.17$ & 0.039 \\
CRP & 1.44 & $1.10-4.53$ & 0.027 \\
\hline
\end{tabular}

ILD, interstitial lung disease; pSS, primary Sjögren's syndrome; $\mathrm{RR}$, relative risk; $\mathrm{Cl}$, confidence interval; NLR, neutrophil to lymphocyte ratio; ALB, albumin; CRP, C-reactive protein.
Table 5 Analysis of prognostic factors related to pSS patients

\begin{tabular}{lccc}
\hline Factors & OR & $95 \% \mathrm{Cl}$ & $\mathrm{P}$ value \\
\hline Age & 1.28 & $1.06-2.25$ & 0.042 \\
NLR & 1.43 & $1.12-2.57$ & 0.034 \\
Irregular treatment & 1.51 & $1.18-3.01$ & 0.030 \\
ILD & 2.05 & $1.36-4.72$ & 0.023 \\
\hline pSS, primary Sjögren's syndrome; OR, odds & ratio; CI, \\
confidence interval; NLR, neutrophil to lymphocyte ratio; ILD, \\
interstitial lung disease.
\end{tabular}

was $13 \%$ [ $95 \%$ confidence interval (CI): $9 \%$ to $19 \%$ ], and the detection rate of Asian patients was higher than that of European patients (20\% vs. $10 \%$ ). The risk factors for ILD in pSS patients include being: male [odds ratio $(\mathrm{OR})=1.92$, 95\% CI: 1.26 to 2.95], elderly [weighted mean difference $(\mathrm{WMD})=9.25$ years, $95 \%$ CI: 2.78 to 15.72 ], and having elevated CRP (WMD =3.92 mg/L, 95\% CI: 0.27 to 1.61 ). The results of this study were inconsistent with the abovementioned factors, especially in that the study did not reveal that the male gender can be a risk factor. It can also be related to different populations and sample sizes. Some studies have found that certain new biomarkers are also related to the occurrence of ILD in patients with pSS, including interleukin-33 (IL-33) and soluble ST2 (24). The serum levels of angiopoietin-like protein 2 and galectin-3 in pSS patients with ILD have been revealed as significantly higher than those in NILD patients $(25,26)$. However, it will take a long time for these biomarkers to be applied in clinical work. This study first analyzed the factors related to the presence of ILD at the first diagnosis of pSS patients, and then followed up NILD participants. It was found that among 105 participants who reviewed HRCT during the follow-up period, 18 were diagnosed with ILD; we further did a multivariate analysis and found that baseline NLR, CRP, age, and non-regular treatment were risk factors for ILD in patients with pSS during follow-up. This result suggests that in clinical practice, if older patients with pSS are found to have higher levels of NLR and CRP at the first diagnosis, it should be noted that the risk of ILD in the future is significantly increased, and if the treatment of pSS is not regular, the risk of ILD will further increase, but there is no clear evidence on whether HRCT should be performed regularly for screening (27). In fact, many patients are currently diagnosed with ILD first, and then 
diagnosed with pSS when investigating the cause (28). For high-risk pSS patients, noninvasive and simple methods can be considered for respiratory function monitoring, such as simple lung function grading $(14,15,29)$, or performing lung function tests to detect impaired small airway ventilation as soon as possible $(30,31)$.

Once ILD occurs in pSS patients, the prognosis is poor, and the 5 -year survival rate is approximately $88.5 \%$ (32). The results of this study are basically consistent with those of previous studies. In this study, 35 participants (16.1\%) died within 1-18 years (median follow-up time was 8 years) follow-up. However, it has also been observed clinically that although HRCT suggests interstitial lesions in some patients, there is no obvious manifestation of decreased lung function during long-term follow-up $(19,33)$. Therefore, it is necessary to further identify those patients who have both imaging pulmonary interstitial changes and high clinical risk. In a study, $\mathrm{Xu}$ et al. found that extensive lung involvement is an independent risk factor for the progression of ILD in patients with pSS, and hypoproteinemia is an independent risk factor for death in such patients (34). In another study conducted by Kamiya et al., the authors found that lower percentage of forced vital capacity and higher serum Krebs von den Lungen-6 (KL-6) levels are predictors of poor prognosis in patients with pSS-ILD (35).

In short, the results of this study initially suggested that NLR, which is easily testable in clinical practice, can be used as a predictor of ILD in patients with pSS, and can also be used as a predictor of long-term follow-up outcome for patients with pSS. The exploration of this potential use of NLR is worthy of further multicenter and prospective research. There were some limitations in this study. First, because it was a single-center study, the sample size was small. Secondly, because it was a retrospective analysis, not every patient diagnosed with pSS could be regularly followed up and undergo HRCT examination during the follow-up period. It is possible that some patients with ILD may have been missed. It is necessary to design a multicentered, prospective study on the basis of expanding the sample size to observe changes in the lung involvement and the prognosis of patients with pSS after the initial diagnosis of pSS.

\section{Acknowledgments}

Funding: None.

\section{Footnote}

Reporting Checklist: The authors have completed the STROBE reporting checklist. Available at http://dx.doi. org/10.21037/apm-21-1043

Data Sharing Statement: Available at http://dx.doi. org/10.21037/apm-21-1043

Conflicts of Interest: All authors have completed the ICMJE uniform disclosure form (available at http://dx.doi. org/10.21037/apm-21-1043). The authors have no conflicts of interest to declare.

Ethical Statement: The authors are accountable for all aspects of the work in ensuring that questions related to the accuracy or integrity of any part of the work are appropriately investigated and resolved. This study was approved by the Ethics Committee of Meizhou People's Hospital (2020-C-102), and the study followed the Declaration of Helsinki (2013 revision). All participants provided written informed consent.

Open Access Statement: This is an Open Access article distributed in accordance with the Creative Commons Attribution-NonCommercial-NoDerivs 4.0 International License (CC BY-NC-ND 4.0), which permits the noncommercial replication and distribution of the article with the strict proviso that no changes or edits are made and the original work is properly cited (including links to both the formal publication through the relevant DOI and the license). See: https://creativecommons.org/licenses/by-nc-nd/4.0/.

\section{References}

1. $\mathrm{Ng} \mathrm{KH}$, Chen DY, Lin CH, et al. Risk of interstitial lung disease in patients with newly diagnosed systemic autoimmune rheumatic disease: a nationwide, populationbased cohort study. Semin Arthritis Rheum 2020;50:840-5.

2. Dong X, Zhou J, Guo X, et al. A retrospective analysis of distinguishing features of chest HRCT and clinical manifestation in primary Sjögren's syndrome-related interstitial lung disease in a Chinese population. Clin Rheumatol 2018;37:2981-8.

3. Gupta S, Ferrada MA, Hasni SA. Pulmonary manifestations of primary Sjögren's syndrome: underlying immunological mechanisms, clinical presentation, and 
management. Front Immunol 2019;10:1327.

4. Amlani B, Elsayed G, Barvalia U, et al. Treatment of primary Sjögren's syndrome-related interstitial lung disease: a retrospective cohort study. Sarcoidosis Vasc Diffuse Lung Dis 2020;37:136-47.

5. Zheng L, Shi Y, Zhi C, et al. Loose combined cutting seton for patients with high intersphincteric fistula: a retrospective study. Ann Transl Med 2020;8:1236.

6. Zhao R, Wang Y, Zhou W, et al. Associated factors with interstitial lung disease and health-related quality of life in Chinese patients with primary Sjögren's syndrome. Clin Rheumatol 2020;39:483-9.

7. Jeny F, Brillet PY, Kim YW, et al. The place of highresolution computed tomography imaging in the investigation of interstitial lung disease. Expert Rev Respir Med 2019;13:79-94.

8. Li L, Xia Y, Chen C, et al. Neutrophil-lymphocyte ratio in systemic lupus erythematosus disease: a retrospective study. Int J Clin Exp Med 2015;8:11026-31.

9. Okba AM, Amin MM, Abdelmoaty AS, et al. Neutrophil/ lymphocyte ratio and lymphocyte/monocyte ratio in ulcerative colitis as non-invasive biomarkers of disease activity and severity. Auto Immun Highlights 2019;10:4.

10. World Medical Association. World Medical Association Declaration of Helsinki: ethical principles for medical research involving human subjects. JAMA 2013;310:2191-4.

11. Hansell DM, Goldin JG, King TE Jr, et al. CT staging and monitoring of fibrotic interstitial lung diseases in clinical practice and treatment trials: a position paper from the Fleischner Society. Lancet Respir Med 2015;3:483-96.

12. Chen L, Halai V, Leandru A, et al. Interstitial lung disease: update on the role of computed tomography in the diagnosis of idiopathic pulmonary fibrosis. J Comput Assist Tomogr 2019;43:898-905.

13. Bradley B, Branley HM, Egan JJ, et al. Interstitial lung disease guideline: the British Thoracic Society in collaboration with the Thoracic Society of Australia and New Zealand and the Irish Thoracic Society. Thorax 2008;63 Suppl 5:v1-58. Erratum in: Thorax 2008;63:1029.

14. Jones NL. New tests to asses lung function. Exercise tesing in pulmonary evaluation: clinical applications. $\mathrm{N}$ Engl J Med 1975;293:647-50.

15. Liang BM, Lam DC, Feng YL. Clinical applications of lung function tests: a revisit. Respirology 2012;17:611-9.

16. Salaffi F, Di Carlo M, Carotti M, et al. The impact of different rheumatic diseases on health-related quality of life: a comparison with a selected sample of healthy individuals using SF-36 questionnaire, EQ-5D and SF-6D utility values. Acta Biomed 2019;89:541-57.

17. Development of the World Health Organization WHOQOL-BREF quality of life assessment. The WHOQOL Group. Psychol Med 1998;28:551-8.

18. Luppi F, Sebastiani M, Silva M, et al. Interstitial lung disease in Sjögren's syndrome: a clinical review. Clin Exp Rheumatol 2020;38 Suppl 126:291-300.

19. Wells AU, Denton CP. Interstitial lung disease in connective tissue disease--mechanisms and management. Nat Rev Rheumatol 2014;10:728-39.

20. Fischer A, du Bois R. Interstitial lung disease in connective tissue disorders. Lancet 2012;380:689-98.

21. Mathai SC, Danoff SK. Management of interstitial lung disease associated with connective tissue disease. BMJ 2016;352:h6819.

22. Wallace B, Vummidi D, Khanna D. Management of connective tissue diseases associated interstitial lung disease: a review of the published literature. Curr Opin Rheumatol 2016;28:236-45.

23. He C, Chen Z, Liu S, et al. Prevalence and risk factors of interstitial lung disease in patients with primary Sjögren's syndrome: a systematic review and meta-analysis. Int J Rheum Dis 2020;23:1009-18.

24. Zhao L, Yao L, Yuan L, et al. Potential contribution of interleukin-33 to the development of interstitial lung disease in patients with primary Sjögren's Syndrome. Cytokine 2013;64:22-4.

25. Shi L, Fu Q, Chen N, et al. Angiopoietin-like protein 2 as a novel marker for patients with primary Sjögren's syndrome-related interstitial lung disease. Clin Exp Med 2020;20:393-9.

26. Zhang R, Sun T, Song L, et al. Increased levels of serum galectin-3 in patients with primary Sjögren's syndrome: associated with interstitial lung disease. Cytokine 2014;69:289-93.

27. Jee AS, Sheehy R, Hopkins P, et al. Diagnosis and management of connective tissue disease-associated interstitial lung disease in Australia and New Zealand: a position statement from the Thoracic Society of Australia and New Zealand. Respirology 2021;26:23-51.

28. Reina D, Roig Vilaseca D, Torrente-Segarra V, et al. Sjögren's syndrome-associated interstitial lung disease: a multicenter study. Reumatol Clin 2016;12:201-5.

29. Carbone RG, Paredi P, Monselise A, et al. New York Heart Association class associated with imaging is a prognostic mortality risk predictor in interstitial lung diseases. Eur Rev Med Pharmacol Sci 2020;24:9012-21. 
30. Nakanishi M, Fukuoka J, Tanaka T, et al. Small airway disease associated with Sjögren's syndrome: clinicopathological correlations. Respir Med 2011;105:1931-8.

31. Papiris SA, Maniati M, Constantopoulos SH, et al. Lung involvement in primary Sjögren's syndrome is mainly related to the small airway disease. Ann Rheum Dis 1999;58:61-4.

32. Gao H, Zhang XW, He J, et al. Prevalence, risk factors, and prognosis of interstitial lung disease in a large cohort of Chinese primary Sjögren syndrome patients: a casecontrol study. Medicine (Baltimore) 2018;97:e11003.

33. Zhang T, Yuan F, Xu L, et al. Characteristics of patients

Cite this article as: Zhang $\mathrm{K}, \mathrm{Li} \mathrm{W}, \mathrm{He} \mathrm{C}, \mathrm{He} \mathrm{X}$, Hou J. The ratio of neutrophil to lymphocyte predicts interstitial pneumonia and its prognosis in patients with primary Sjögren's syndrome: a retrospective analysis. Ann Palliat Med 2021;10(6):6493-6501. doi: 10.21037/apm-21-1043 with primary Sjögren's syndrome associated interstitial lung disease and relevant features of disease progression. Clin Rheumatol 2020;39:1561-8.

34. Xu Y, Zhou J, Dong X, et al. Risk factors for progression and prognosis of primary Sjögren's syndrome-associated interstitial lung disease in a Chinese population. Int J Rheum Dis 2020;23:1734-40.

35. Kamiya Y, Fujisawa T, Kono M, et al. Prognostic factors for primary Sjögren's syndrome-associated interstitial lung diseases. Respir Med 2019;159:105811.

(English Language Editor: J. Jones) 